\title{
Analyses of risk factors for polycystic ovary syndrome complicated with non-alcoholic fatty liver disease
}

\author{
JIANHAI ZHANG ${ }^{*}$, JIAN HU* ${ }^{*}$ CHUNXIA ZHANG, YANNI JIAO, XIANG KONG and WEI WANG \\ Department of Obstetrics and Gynecology, Shengli Oilfield Central Hospital, Dongying, Shandong 257034, P.R. China
}

Received December 18, 2017; Accepted February 15, 2018

DOI: $10.3892 /$ etm.2018.5932

\begin{abstract}
The risk factors related to polycystic ovary syndrome (PCOS) patients complicated with non-alcoholic fatty liver disease (NAFLD) were investigated. A total of 188 PCOS patients treated in Shengli Oilfield Central Hospital (Dongying, China) from February 2014 to February 2015 were retrospectively analyzed as PCOS group, and PCOS group was further divided into NAFLD group and non-NAFLD (N-NAFLD) group according to the liver B ultrasound. In the same time-period, 65 healthy people were selected as normal control group. The differences of clinical, biochemical and metabolic indexes were compared. The levels of luteinizing hormone ( $\mathrm{LH}), \mathrm{LH} /$ follicle stimulating hormone (FSH), testosterone $(\mathrm{T})$, free androgen index (FAI), fasting insulin (FINS) and homeostasis model assessment of insulin resistance (HOMA-IR) index in PCOS group were higher than those in normal control group, but the sex hormone binding globulin (SHBG) level was lower than that in normal control group $(\mathrm{P}<0.05)$; there were no statistically significant differences in comparisons of age, body mass index (BMI), waist-hip ratio (WHR), FSH, dehydroepiandrosterone sulfate (DHEAs) and fasting blood glucose (FBG) between the two groups $(\mathrm{P}>0.05)$. The prevalence rate of NAFLD in PCOS group $(44.68 \%)$ was significantly higher than that in control group $(24.62 \%)$ $(\mathrm{P}<0.05)$. The proportion of NAFLD in PCOS patients in obesity group $(63.51 \%)$ was significantly higher than that in non-obesity group (15.79\%) $(\mathrm{P}<0.05)$. In PCOS group, NAFLD patients had more obvious metabolic abnormalities [high BMI, WHR, FBG, FINS, HOMA-IR index, total cholesterol (TC) and triglyceride (TG), and low high-density lipoprotein HDL and SHBG] and androgen excess compared with those in N-NAFLD patients $(\mathrm{P}<0.05)$. The levels of $\mathrm{LH}, \mathrm{LH} / \mathrm{FSH}$,
\end{abstract}

Correspondence to: Dr Wei Wang, Department of Obstetrics and Gynecology, Shengli Oilfield Central Hospital, 31 Jinan Road, Dongying, Shandong 257034, P.R. China

E-mail:weq3s8@163.com

*Contributed equally

Key words: mitral valve replacement, pulmonary infection, metabolic syndrome, blood glucose, blood lipid
FINS and HOMA-IR index in PCOS group complicated with NAFLD were higher than those in control group complicated with NAFLD $(\mathrm{P}<0.05)$, but the differences in age, BMI, WHR, FSH and FBG levels were not statistically significant between the two groups $(\mathrm{P}>0.05)$. HOMA-IR index, BMI, WHR and TG were independent risk factors for PCOS complicated with NAFLD $(\mathrm{P}<0.05)$.

\section{Introduction}

Polycystic ovary syndrome (PCOS) is a common difficult and complicatedgynecologic disease, which often occurs in puberty and childbearing period, affecting 6-21\% women (1). It is mainly characterized by anovulation or rare ovulation, clinical or biochemical androgen excess and polycystic ovary, and insulin resistance (IR) is an important pathophysiological change (2). Due to long-term metabolic disorders, the middle-aged and elderly people suffer from diabetes mellitus, cardiovascular diseases and even tumors, which will affect their whole lives if treated improperly. Studies have found that non-alcoholic fatty liver disease (NAFLD) is closely related to obesity, abnormal glucose tolerance, IR, dyslipidemia and other factors (3). Both PCOS and NAFLD are diseases with IR as the core pathogenesis, and previous studies have suggested there is a correlation between them $(4,5)$. Some scholars believe that NAFLD may be one of the complications of PCOS. The correlation between PCOS and NAFLD is a research hotspot at present. In this study, the risk factors for PCOS complicated with NAFLD were analyzed, so as to provide clinical references for the early diagnosis and treatment of PCOS patients complicated with NAFLD.

\section{Patients and methods}

Clinical data. According to the unified standard developed in Rotterdam International Conference in 2003 (6), 188 PCOS patients with complete clinical data, admitted into the Department of Obstetrics and Gynecology, Shengli Oilfield Central Hospital (Dongying, China) from February 2014 to February 2015, were collected as PCOS group. A total of 65 healthy outpatients receiving physical examination during the same period were collected as normal control group. Inclusion criteria of normal group: i) patients with regular menstruation and without biochemical and clinical androgen excess; ii) patients without history of endocrine diseases; 
iii) patients without polycystic manifestations in bilateral ovaries via B ultrasound. Exclusion criteria: i) patients complicated with other endocrine diseases (Cushing syndrome, thyroid dysfunction and premature ovarian failure); ii) patients with smoking history, alcohol abuse history, hypertension or coronary heart disease history; iii) patients with history of diseases in important organs, such as heart, liver or kidney; iv) patients who received drug therapy for PCOS in the past 3 months or took drugs affecting the glucose and lipid metabolism (such as glucocorticoids); v) patients with history of chronic liver disease (viral hepatitis, alcoholic hepatitis or other liver diseases). Diagnostic criteria revised in the Guideline (2010) were used for NAFLD. This study obtained ethics approval from the Ethics Committee of Shengli Oilfield Central Hospital, and patients were informed and signed the consent.

\section{Research methods}

General clinical data. General clinical data of the patients, including the name, age, history of menstruation, history of marriage and childbearing, smoking history, drinking history, previous history (hypertension, diabetes mellitus, coronary heart disease, pituitary disease, fatty liver, viral hepatitis), family history and medication, were recorded.

Anthropometric parameters. The weight of the participants in fasting state and single-layer garment was measured, the waist circumference and hip circumference were measured under stable breathing, and the height was measured. Each index was measured twice and the average was taken. Body mass index $(\mathrm{BMI})=$ height $(\mathrm{kg}) /$ weight $\left(\mathrm{m}^{2}\right)$, and $\mathrm{BMI} \geq 25$ indicated obesity; waist-hip ratio (WHR) = waist circumference/hip circumference.

Detection of serological indexes. Specimens were collected from all objects on the 3rd-5th days in menstruation. At $8 \mathrm{~h}$ after fasting, antecubital venous blood was drawn from the patients in quiet and fasting states in the morning, and detected as soon as possible. The levels of follicle-stimulating hormone (FSH), luteinizing hormone (LH), prolactin (PRL), estradiol (E2) and testosterone (T) were detected via immunochemiluminescence method. Serum dehydroepiandrosterone sulfate (DHEAs), sex hormone binding globulin (SHBG) and alanine aminotransferase (ALT) were detected via double antibody sandwich enzyme-linked immunosorbent assay (ELISA). Serum triglyceride (TG), total cholesterol (TC), high-density lipoprotein (HDL), low-density lipoprotein (LDL) and fasting blood glucose (FBG) were detected using the enzymatic method. Moreover, fasting insulin (FINS) was detected via radioimmunoassay, and homeostasis model assessment (HOMA) was used for the evaluation of insulin resistance (IR); HOMA-IR index $=[\mathrm{FBG}(\mathrm{mmol} / \mathrm{l}) \times$ FINS $(\mathrm{mU} / \mathrm{l})] / 22.5$. Free androgen index $(\mathrm{FAI})=\mathrm{T}(\mathrm{nmol} / \mathrm{l}) \times \mathrm{100} / \mathrm{SHBG}(\mathrm{nmol} / \mathrm{l})$. All operations were performed by experts in strict accordance with the instructions.

Vaginal ultrasound examination. All the patients received vaginal ultrasound examination to detect the antral follicle count (AFC) and bilateral ovarian volume, and they all underwent abdominal liver B ultrasound examination to exclude measurement errors. Each index was measured twice.
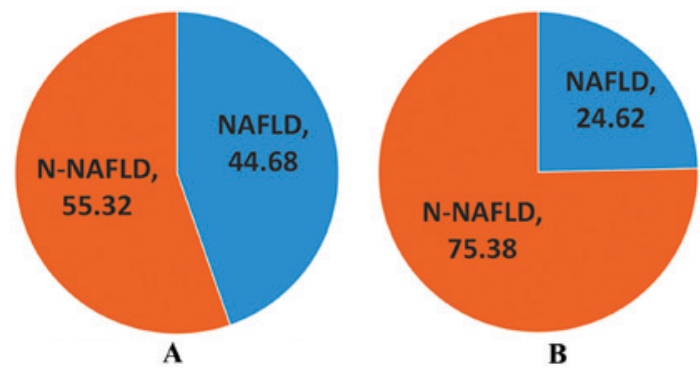

Figure 1. Prevalence rates of NAFLD. (A) Prevalence rate of NAFLD in normal control group (\%); (B) Prevalence rate of NAFLD in PCOS group (\%).

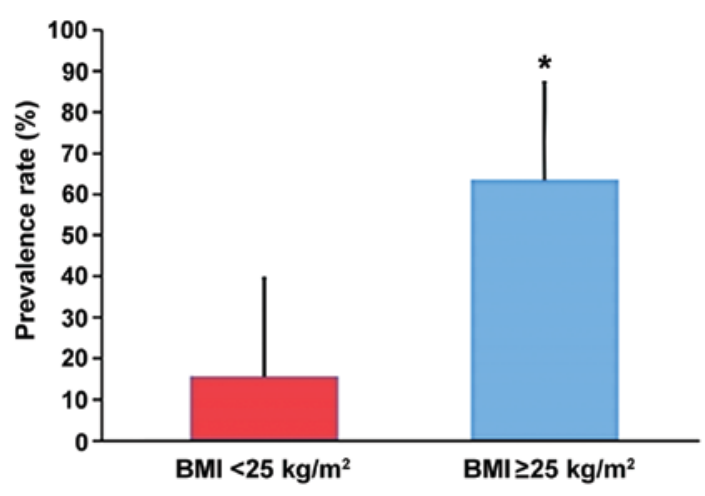

Figure 2. Prevalence rate of NAFLD in patients with different BMI in PCOS group. BMI $<25 \mathrm{~kg} / \mathrm{m}^{2}$ vs. $\geq 25 \mathrm{~kg} / \mathrm{m}^{2}$ group, ${ }^{*} \mathrm{P}<0.05$.

Statistical analysis. Data were recorded using SPSS 20.0 software (SPSS Inc., Chicago, IL, USA). Measurement data are presented as (mean $\pm \mathrm{SD}$ ) and t-test was used for comparison. Measurement data in abnormal distribution are presented as mean value $[95 \%$ confidence interval $(\mathrm{CI})]$, and rank sum test was used for comparison. Logistic regression analysis was used for the multivariate result analysis. $\mathrm{P}<0.05$ indicated that the difference was statistically significant.

\section{Results}

Comparison of clinical data of patients between control group and PCOS group. There were no statistically significant differences in comparisons of age, BMI, WHR, FSH, DHEA and FBG between the two groups ( $\mathrm{P}>0.05)$. The levels of $\mathrm{LH}$, LH/FSH, T, FAI, FINS and HOMA-IR index in PCOS group were higher than those in normal control group $(\mathrm{P}<0.05)$, but the SHBG level was lower than that in normal control group $(\mathrm{P}<0.05)$, as shown in Table I.

Comparison of prevalence rate of NAFLD between normal control group and PCOS group. There were 16 cases (24.62\%) of NAFLD in normal group, and 84 cases (44.68\%) of NAFLD in PCOS group. The prevalence rate of NAFLD in PCOS group was significantly higher than that in normal control group $(\mathrm{P}<0.05)$ (Fig. 1).

Comparison of prevalence rate of NAFLD in patients with different BMI in PCOS group. Among 188 PCOS patients, there were 74 cases with BMI $\geq 25$ (obesity group) and 114 cases 
Table I. Comparisons of clinical data of patients between control group and PCOS group.

\begin{tabular}{lccc}
\hline Index & Control group $(\mathrm{n}=65)$ & PCOS group $(\mathrm{n}=188)$ & P-value \\
\hline Age $($ years $)$ & $26.96 \pm 4.78$ & $27.14 \pm 5.23$ & 0.056 \\
BMI $\left(\mathrm{kg} / \mathrm{m}^{2}\right)$ & $24.23 \pm 2.97$ & $25.18 \pm 3.21$ & 0.124 \\
WHR & $0.87 \pm 0.75$ & $0.89 \pm 0.82$ & 0.573 \\
LH $(\mathrm{IU} / \mathrm{l})$ & $4.52 \pm 2.25$ & $8.10 \pm 2.76$ & $>0.05$ \\
FSH $(\mathrm{IU} / \mathrm{l})$ & $6.73 \pm 2.85$ & $6.16 \pm 3.12$ & 0.212 \\
LH/FSH & $0.67 \pm 0.22$ & $1.31 \pm 0.28$ & $>0.05$ \\
T $(\mathrm{nmol} / \mathrm{l})$ & $1.69 \pm 0.58$ & $2.19 \pm 0.88$ & $>0.05$ \\
DHEA $(\mu \mathrm{g} / \mathrm{dl})$ & $215.86 \pm 84.37$ & $225.37 \pm 101.69$ & 0.975 \\
SHBG $(\mathrm{nmol} / \mathrm{l})^{\mathrm{a}}$ & $45.12(37.54-50.87)$ & $34.45(28.68-40.13)$ & 0.001 \\
FAI & $4.67(3.85-5.59)$ & $10.82(8.89-12.51)$ & $>0.05$ \\
FBG $(\mathrm{mmol} / \mathrm{l})$ & $4.83 \pm 0.41$ & $4.85 \pm 0.46$ & 0.659 \\
FINS $(\mathrm{mIU} / \mathrm{l})^{\mathrm{a}}$ & $9.92(8.50-11.42)$ & $15.12(12.56-17.39)$ & $3.53 \pm 0.64$ \\
HOMA-IR & $1.56 \pm 0.28$ & & $>0.024$ \\
\hline
\end{tabular}

aNon-normal data.

Table II. Comparisons of clinical data between NAFLD group and N-NAFLD group in PCOS group.

\begin{tabular}{lccc}
\hline Index & N-NAFLD group $(\mathrm{n}=104)$ & NAFLD group $(\mathrm{n}=84)$ & P-value \\
\hline Age $($ years $)$ & $25.16 \pm 5.07$ & $27.32 \pm 6.15$ & 0.007 \\
BMI $\left(\mathrm{kg} / \mathrm{m}^{2}\right)$ & $23.25 \pm 3.18$ & $28.61 \pm 4.27$ & $>0.05$ \\
WHR & $0.83 \pm 0.09$ & $10.56 \pm 0.08$ & $>0.05$ \\
LH $(\mathrm{IU} / \mathrm{l})$ & $8.41 \pm 5.36$ & $7.13 \pm 3.09$ & 0.061 \\
FSH $(\mathrm{IU} / \mathrm{l})$ & $7.36 \pm 2.79$ & $1.51 \pm 1.14$ & 0.523 \\
LH/FSH & $1.23 \pm 0.82$ & $2.17 \pm 0.89$ & 0.109 \\
T $(\mathrm{nmol} / \mathrm{l})$ & $2.19 \pm 0.78$ & $1.66 \pm 0.22$ & 0.513 \\
TG $(\mathrm{mmol} / \mathrm{l})$ & $0.93 \pm 0.14$ & $4.78 \pm 0.91$ & $>0.05$ \\
TC $(\mathrm{mmol} / \mathrm{l})$ & $4.38 \pm 1.04$ & $1.14 \pm 0.21$ & 0.029 \\
HDL $(\mathrm{mmol} / \mathrm{l})$ & $1.54 \pm 0.39$ & $219.75 \pm 98.46$ & $>0.05$ \\
DHEAs $(\mu \mathrm{g} / \mathrm{dl})$ & $228.56 \pm 111.84$ & $13.97(11.61-16.37)$ & 0.478 \\
SHBG $(\mathrm{nmol} / \mathrm{l})^{\mathrm{a}}$ & $49.21(40.47-57.69)$ & $5.02 \pm 0.46$ & $>0.05$ \\
FAI & $6.71(5.35-7.98)$ & $19.71(16.56-22.82)$ & $>0.05$ \\
FBG $(\mathrm{mmol} / \mathrm{l})$ & $4.74 \pm 0.43$ & $4.83 \pm 0.84$ & 0.002 \\
FINS $(\mathrm{mIU} / \mathrm{l})^{\mathrm{a}}$ & $8.79(7.45-10.14)$ & $42.12(35.04-49.20)$ & $>0.05$ \\
HOMA-IR & $1.90 \pm 0.32$ & $>0.05$ \\
ALT $(\mathrm{U} / \mathrm{l})^{\mathrm{a}}$ & $13.37(11.26-15.48)$ & $>0.05$ \\
\hline
\end{tabular}

${ }^{\mathrm{a} N o n-n o r m a l ~ d a t a . ~}$

with BMI <25 (non-obesity group). The prevalence rate of NAFLD in PCOS patients in obesity group (47/74, 63.51\%) was higher than that in PCOS patients in non-obesity group (18/114, $15.79 \%)\left(\chi^{2}=30.12\right)$ (Fig. 2).

Comparisons of clinical data between NAFLD group and $N$-NAFLD group in PCOS group. NAFLD group had higher age, BMI and WHR than N-NAFLD group $(\mathrm{P}<0.05)$; the FAI level in NAFLD group was higher than that in N-NAFLD group, but the SHBG level in NAFLD group was lower than that in N-NAFLD group $(\mathrm{P}<0.05)$; there were no statistically significant differences in comparisons of T, LH, FSH and LH/FSH between the two groups ( $\mathrm{P}>0.05)$; the levels of FBG, FINS and HOMA-IR index in NAFLD group were higher than those in N-NAFLD group $(\mathrm{P}<0.05)$; the levels of TC and TG in NAFLD group were higher than those in N-NAFLD 
Table III. Comparisons of clinical data between PCOS group complicated with NAFLD and control group complicated with NAFLD.

\begin{tabular}{lccr}
\hline Index & Control group complicated with NAFLD $(\mathrm{n}=16)$ & PCOS group complicated with NAFLD $(\mathrm{n}=84)$ & P-value \\
\hline Age $(\mathrm{years})$ & $27.46 \pm 4.49$ & $27.32 \pm 6.15$ & 0.057 \\
BMI $\left(\mathrm{kg} / \mathrm{m}^{2}\right)$ & $28.92 \pm 3.68$ & $28.61 \pm 4.27$ & 0.192 \\
WHR & $0.91 \pm 0.07$ & $0.94 \pm 0.08$ & 0.936 \\
LH $(\mathrm{IU} / \mathrm{l})$ & $8.41 \pm 5.36$ & $10.56 \pm 9.05$ & 0.061 \\
FSH $(\mathrm{IU} / \mathrm{l})$ & $7.36 \pm 2.79$ & $7.13 \pm 3.09$ & 0.523 \\
LH/FSH & $1.23 \pm 0.82$ & $1.51 \pm 1.14$ & 0.109 \\
T $(\mathrm{nmol} / \mathrm{l})$ & $1.66 \pm 0.93$ & $2.17 \pm 0.89$ & $>0.05$ \\
DHEAs $(\mu \mathrm{g} / \mathrm{dl})$ & $217.43 \pm 115.31$ & $219.75 \pm 98.46$ & 0.962 \\
SHBG $(\mathrm{nmol} / \mathrm{l})^{\mathrm{a}}$ & $39.89(22.65-43.13)$ & $24.16(18.98-29.34)$ & 0.002 \\
FAI & $6.35(4.31-8.39)$ & $13.97(11.61-16.37)$ & $>0.05$ \\
FBG $(\mathrm{mmol} / \mathrm{l})$ & $4.84 \pm 0.43$ & $5.02 \pm 0.46$ & 0.831 \\
FINS $(\mathrm{mIU} / \mathrm{l})^{\mathrm{a}}$ & $13.21(11.05-15.38)$ & $19.71(16.56-22.82)$ & 0.027 \\
HOMA-IR & $2.30 \pm 0.62$ & $4.83 \pm 0.84$ & $>0.05$ \\
\hline
\end{tabular}

${ }^{\mathrm{a} N o n-n o r m a l ~ d a t a . ~}$

Table IV. Logistic regression analyses of influencing factors of PCOS complicated with NAFLD.

\begin{tabular}{lcccc}
\hline Factors & b-value & Wald $\chi^{2}$ value & P-value & OR (95\% CI) \\
\hline HOMA-IR & 3.109 & 32.324 & 0.001 & $28.903(7.835-65.641)$ \\
BMI & 2.184 & 16.672 & 0.002 & $21.542(4.352-47.436)$ \\
TG & 0.523 & 4.839 & 0.026 & $1.685(1.055-2.693)$ \\
WHR & 0.531 & 1.643 & 0.045 & $1.712(0.006-2.894)$ \\
\hline
\end{tabular}

group $(\mathrm{P}<0.05)$, but the level of HDL was lower than that in N-NAFLD group $(\mathrm{P}<0.05)$; the level of ALT in NAFLD group was higher than that in N-NAFLD group $(\mathrm{P}<0.05)$ (Table II).

Comparisons of clinical data between PCOS group complicated with NAFLD and control group complicated with $N A F L D$. There were no statistically significant differences in comparisons of age, BMI and WHR between PCOS group complicated with NAFLD and control group complicated with NAFLD $(\mathrm{P}>0.05)$; LH and LH/FSH in PCOS group complicated with NAFLD were higher than those in control group complicated with NAFLD $(\mathrm{P}<0.05)$, but there was no statistically significant difference in the FSH level between the two groups $(\mathrm{P}>0.05)$; compared with those in control group complicated with NAFLD, FINS and HOMA-IR index in PCOS group complicated with NAFLD were higher $(\mathrm{P}<0.05)$, but there was no statistically significant difference in the FBG level between the two groups $(\mathrm{P}>0.05)$ (Table III).

Logistic regression analyses of influencing factors of PCOS complicated with NAFLD. HOMA-IR index [odds ratio $(\mathrm{OR})=28.903]$, BMI $(\mathrm{OR}=21.542)$, WHR $(\mathrm{OR}=1.712)$ and $\mathrm{TG}$ $(\mathrm{OR}=1.685)$ were risk factors for PCOS complicated with NAFLD. HOMA-IR had the highest correlation with PCOS complicated with NAFLD, followed by BMI, WHR and TG. The higher the HOMA-IR index, BMI, WHR and TG levels were, the higher the risk of PCOS in NAFLD patients would be (Table IV).

\section{Discussion}

Our results showed that the prevalence rate of PCOS complicated with NAFLD was $44.68 \%$, which is consistent with previous reports (7); it was significantly higher than the prevalence rate of NAFLD in normal people $(24.62 \%, \mathrm{P}<0.05)$. It has been shown that obesity is the most important risk factor for NAFLD (8). Obesity is also the most common clinical manifestation of PCOS patients, and approximately 2/3-4/5 PCOS patients suffer from obesity (9). It was also found in this study that NAFLD patients in PCOS group had higher BMI than N-NAFLD patients, which is consistent with the results of the study by Kauffman et al (10). BMI $\geq 25$ indicates obesity; the prevalence rate of NAFLD in obesity patients with PCOS (63.51\%) was significantly higher than that in non-obesity patients $(15.79 \%)(\mathrm{P}<0.05)$, suggesting that obesity is closely related to PCOS complicated with NAFLD. In the present study the incidence rate of transaminase disorder in PCOS patients complicated with NAFLD was higher. Besides, studies have shown that liver cells with fatty degeneration more or less 
leads to impaired liver function (11), but another study showed that more than $70 \%$ people with normal liver enzyme have been in various stages of NAFLD, among which $1 / 3$ people showed fibrosis, and 1/10 people show liver cirrhosis (12). B ultrasound is simple, economical and non-invasive with good reproducibility, good sensitivity and high specificity, so it is more widely accepted than pathological examination of liver tissues $(13,14)$, and has become an important method for the diagnosis and monitoring of NAFLD. PCOS patients with normal liver function need to be paid close attention to. Serum ALT is a sensitive index of liver injury and liver fibrosis, and an important basis of NAFLD clinical typing (15). Clinically, PCOS patients should receive liver ultrasound examination regularly, so as to realize the early detection and dynamic monitoring of fatty liver. In particular, the examination of obesity patients needs to be strengthened; liver function examination is of significance in the active treatment of NAFLD.

At present, it is generally accepted that the mechanism of NAFLD is the 'second-strike' hypothesis (16). The IR-induced lipid accumulation in hepatocytes is the 'first strike' against the liver, and the oxidative stress-induced inflammation and apoptosis is the 'second strike', resulting in gene regulation imbalance of intrahepatic lipid synthesis and fatty acid oxidation. IR is defined as a biological effect of lower insulin than normal level after a certain amount of insulin specifically binds to receptors. NAFLD and IR interact as both cause and effect; on the one hand, NAFLD significantly aggravates the liver IR, increasing risk of type 2 diabetes mellitus (17); on the other hand, a study showed (18) that NAFLD is a result of IR-induced degeneration and necrosis of hepatocytes. It is reported that non-alcoholic steatohepatitis is found in liver biopsy of PCOS patients complicated with IR, and the pathological features of liver tissues are also improved after patients undergo life intervention therapy (19). Increased FINS and HOMA-IR are important indexes of IR. In the present study, the related pathogenic factors to NAFLD in PCOS patients were analyzed. Results showed that compared with those in N-NAFLD patients, BMI, HOMA-IR index, TG, TC, LDL and FAI levels were higher in NAFLD patients in PCOS group, but HDL and SHBG levels were lower $(\mathrm{P}<0.05)$, which is consistent with the conclusion in literature that obesity (20), IR, high TC and low HDL (21) are predisposing factors of NAFLD. IR and androgen excess are two major pathological features of PCOS. This study found that there was a positive correlation between FBG level and androgen level in PCOS patients, and NAFLD patients had more obvious disorders of glucose metabolism indexes (high FBG, FINS and HOMA-IR index) than N-NAFLD patients in PCOS group, and FAI was increased, but SHBG was decreased $(\mathrm{P}<0.05)$; the above findings are similar to the results of Vassilatou et al (22).

In conclusion, analyses of risk factors for PCOS complicated with NAFLD show that HOMA-IR index, BMI, WHR and TG are independent risk factors for PCOS complicated with NAFLD, suggesting that blocking the high risk factors through weight control, avoidance of excessive intake of highfat diet, improvement of IR, increase of insulin sensitivity, is an important link to prevent the occurrence of NAFLD.

\section{Acknowledgements}

Not applicable.

\section{Funding}

No funding was received.

\section{Availability of data and materials}

The datasets used and/or analyzed during the current study are available from the corresponding author on reasonable request.

\section{Authors' contributions}

JZ designed the study and drafted the manuscript. JZ and WW revised the manuscript critically for important intellectual content. JH, CZ and WW acquired the data, analyzed and interpreted statistical analysis. YJ and XK analyzed the data. All authors read and approved the final manuscript and agree to be accountable for all aspects of the study.

\section{Ethics approval and consent to participate}

This study was approved by the Ethics Committee of Shengli Oilfield Central Hospital (Dongying, China). Patients who participated in this research signed the informed consent.

\section{Consent for publication}

Not applicable.

\section{Competing interests}

The authors declare that they have no competing interests.

\section{References}

1. Joham AE, Teede HJ, Ranasinha S, Zoungas S and Boyle J: Prevalence of infertility and use of fertility treatment in women with polycystic ovary syndrome: Data from a large communitybased cohort study. J Womens Health (Larchmt) 24: 299-307, 2015.

2. Rotterdam ESHRE/ASRM-Sponsored PCOS consensus workshop group: Revised 2003 consensus on diagnostic criteria and long-term health risks related to polycystic ovary syndrome (PCOS). Hum Reprod 19: 41-47, 2004.

3. Brunt EM: Nonalcoholic steatohepatitis: Definition and pathology. Semin Liver Dis 21: 3-16, 2001.

4. Kelley CE, Brown AJ, Diehl AM and Setji TL: Review of nonalcoholic fatty liver disease in women with polycystic ovary syndrome. World J Gastroenterol 20: 14172-14184, 2014.

5. Gambarin-Gelwan M, Kinkhabwala SV, Schiano TD, Bodian C, Yeh HC and Futterweit W: Prevalence of nonalcoholic fatty liver disease in women with polycystic ovary syndrome. Clin Gastroenterol Hepatol 5: 496-501,2007.

6. Matthews DR, Hosker JP, Rudenski AS, Naylor BA, Treacher DF and Turner RC: Homeostasis model assessment: Insulin resistance and beta-cell function from fasting plasma glucose and insulin concentrations in man. Diabetologia 28: 412-419, 1985.

7. Brzozowska MM, Ostapowicz G and Weltman MD: An association between non-alcoholic fatty liver disease and polycystic ovarian syndrome. J Gastroenterol Hepatol 24: 243-247.2009.

8. Ortiz-Lopez C, Lomonaco R, Orsak B, Finch J, Chang Z, Kochunov VG, Hardies J and Cusi K: Prevalence of prediabetes and diabetes and metabolic profile of patients with nonalcoholic fatty liver disease (NAFLD). Diabetes Care 35: 873-878, 2012.

9. Teede HJ, Meyer C, Hutchison SK, Zoungas S, McGrath BP and Moran LJ: Endothelial function and insulin resistance in polycystic ovary syndrome: The effects of medical therapy. Fertil Steril 93: 184-191, 2010. 
10. Kauffman RP, Baker TE, Baker V, Kauffman MM and Castracane VD: Endocrine factors associated with non-alcoholic fatty liver disease in women with polycystic ovary syndrome: Do androgens play a role? Gynecol Endocrinol 26: 39-46, 2010.

11. Setji TL, Holland ND, Sanders LL, Pereira KC, Diehl AM and Brown AJ: Nonalcoholic steatohepatitis and nonalcoholic Fatty liver disease in young women with polycystic ovary syndrome. J Clin Endocrinol Metab 91: 1741-1747, 2006.

12. Sorrentino P, Tarantino G, Conca P, Perrella A, Terracciano ML, Vecchione R, Gargiulo G, Gennarelli N and Lobello R: Silent non-alcoholic fatty liver disease-a clinical-histological study. J Hepatol 41: 751-757, 2004.

13. Omagari K, Kadokawa Y, Masuda J, Egawa I, Sawa T, Hazama H, Ohba K, Isomoto H, Mizuta Y, Hayashida K, et al: Fatty liver in non-alcoholic non-overweight Japanese adults: Incidence and clinical characteristics. J Gastroenterol Hepatol 17: 1098-1105, 2002.

14. Xia MF, Yan HM, He WY, Li XM, Li CL, Yao XZ, Li RK, Zeng MS and Gao X: Standardized ultrasound hepatic/renal ratio and hepatic attenuation rate to quantify liver fat content: An improvement method. Obesity (Silver Spring) 20: 444-452, 2012.

15. Gramlich T, Kleiner DE, McCullough AJ, Matteoni CA, Boparai N and Younossi ZM: Pathologic features associated with fibrosis in nonalcoholic fatty liver disease. Hum Pathol 35: 196-199, 2004

16. Menzaghi C, Trischitta V and Doria A: Genetic influences of adiponectin on insulin resistance, type 2 diabetes, and cardiovascular disease. Diabetes 56: 1198-1209, 2007.

17. Choi JH, Rhee EJ, Bae JC, Park SE, Park CY, Cho YK, Oh KW, Park SW and Lee WY: Increased risk of type 2 diabetes in subjects with both elevated liver enzymes and ultrasonographically diagnosed nonalcoholic fatty liver disease: A 4-year longitudinal study. Arch Med Res 44: 115-120, 2013.
18. Marchesini G, Avagnina S, Barantani EG, Ciccarone AM, Corica F, Dall'Aglio E, Dalle Grave R, Morpurgo PS, Tomasi F and Vitacolonna E: Aminotransferase and gamma-glutamyltranspeptidase levels in obesity are associated with insulin resistance and the metabolic syndrome. J Endocrinol Invest 28: 333-339, 2005.

19. Brown AJ, Tendler DA, McMurray RG and Setji TL: Polycystic ovary syndrome and severe nonalcoholic steatohepatitis: Beneficial effect of modest weight loss and exercise on liver biopsy findings. Endocr Pract 11: 319-324, 2005.

20. Lee S, Jin Kim Y, Yong Jeon T, Hoi Kim H, Woo Oh S, Park Y and Soo Kim S: Obesity is the only independent factor associated with ultrasound-diagnosed non-alcoholic fatty liver disease: A cross-sectional case-control study. Scand J Gastroenterol 41: 566-572, 2006.

21. Ohashi K, Ouchi N, Kihara S, Funahashi T, Nakamura T, Sumitsuji S, Kawamoto T, Matsumoto S, Nagaretani H, Kumada M, et al: Adiponectin I164T mutation is associated with the metabolic syndrome and coronary artery disease. J Am Coll Cardiol 43: 1195-1200, 2004

22. Vassilatou E, Lafoyianni S, Vryonidou A, Ioannidis D, Kosma L, Katsoulis K, Papavassiliou E and Tzavara I: Increased androgen bioavailability is associated with non-alcoholic fatty liver disease in women with polycystic ovary syndrome. Hum Reprod 25: 212-220, 2010.

This work is licensed under a Creative Commons Attribution-NonCommercial-NoDerivatives 4.0 International (CC BY-NC-ND 4.0) License. 